\title{
Orthonormal Series Expansion and Laguerre-Finite Hankel Transform of Generalized Functions
}

\author{
S.K.Panchal, \\ Department of Mathematics,Dr.Babasaheb Ambedkar Marathwada University, \\ Aurangabad.PIN-431004,(M.S.) India.E-mail: drpanchalsk@gmail.com
}

\begin{abstract}
In this paper a complete orthonormal family of Laguerre-Bessel functions is derived and certain spaces of testing functions and generalized functions are defined, whose members can be expressed in terms of a Fourier-Laguerre-Bessel series, which gives the inversion formula for Laguerre-Finite Hankel transform of generalized functions. The convergence of the series is interpreted in the weak distributional sense. An operational transform formula is derived which together with the inversion formula is applied in solving certain distributional differential equations.

AMS Subject Classification: $\quad$ 44F12,44A15,46F10,41A58.
\end{abstract}

Key Words:Laguerre-Finite Hankel transform, Fourier-Laguerre-Bessel series, orthonormal series expansion of generalized functions.

\section{Introduction}

Several authors treated the problem of expanding the elements of a distribution space using different orthonormal systems.

Zemanian [1], [5] constructed the testing function space A for suitable complete orthonormal system $\left\{\Psi_{n}\right\}$ of eigenfunctions of the differential operator $\eta$. The elements of the dual space $A^{\prime}$ are generalized functions, each of which can be expanded into a series of eigenfunctions $\Psi_{n}$. As a special case of his general theory he defined the finite Fourier, Hermite, Jacobi and finite Hankel transformations of generalized functions where the inverse transformations are obtained by using orthonormal series expansion of generalized functions.

Bhosale and More [2], Panchal and More [3] extended certain finite integral transformations to generalized functions by using the method of Zemanian. In [4] the author has extended the Laguerre-finite Hankel transform to generalized functions using the Hilbert space technique. In this paper we define Laguerrefinite Hankel transformation and extend it to certain space of generalized functions whose inverse is obtained in terms of Fourier-Laguerre-Bessel series.

\section{Preliminary Results, Notations and Terminology}

Let $\mathrm{I}=\{(\mathrm{x}, \mathrm{t}) / \mathrm{x} \in(0,1), \mathrm{t} \in(0, \infty)\}$ and $\mathrm{N}_{0}=\mathrm{N} \cup\{0\}$. For $v \geq-1 / 2$, let the self adjoint differential operator $\eta_{x, t}=\left(x^{-v-(1 / 2)} D_{x} x^{2 v+1} D_{x} x^{-v-(1 / 2)}\right)\left(e^{t / 2} D_{t} t^{-t} D_{t} e^{t / 2}\right)$ denote the conventional or generalized partial differential operators, where $\mathrm{D}_{\mathrm{x}}=\partial / \partial \mathrm{x}$ and $\mathrm{D}_{\mathrm{t}}=\partial / \partial \mathrm{t}$.

Let $J_{v}(x)$ be the Bessel function of first kind of order $v \geq(-1 / 2)$ and $C^{2}{ }_{m, v}=\left(J^{2}{ }_{v+1}\left(j_{m}\right)\right) / 2$, wherej ${ }_{m}$ are the positive roots of the $J_{v}(j z)=0$ arranged in ascending order of magnitude. Let $L_{n}(t)$ be the Laguerre polynomial of degree $\mathrm{n}$. Consider the eigenfunction system $\left\{\psi_{\mathrm{m}, \mathrm{n}}(\mathrm{x}, \mathrm{t})\right\}_{\mathrm{m}, \mathrm{n}=0}{ }^{\infty}$ corresponding to the differential operatorn $(\mathrm{x}, \mathrm{t})$ where

$$
\psi_{m, n}(x, t)=\frac{\sqrt{x} e^{-t / 2}}{n ! C_{m, \nu}} J_{\nu}\left(j_{m} x\right) L_{n}(t), \quad n, m \in N_{0} .
$$

We see that,

$$
\eta_{\mathrm{x}, \mathrm{t}} \psi_{\mathrm{m}, \mathrm{n}}(\mathrm{x}, \mathrm{t})=\lambda_{\mathrm{m}, \mathrm{n}} \psi_{\mathrm{m}, \mathrm{n}}(\mathrm{x}, \mathrm{t})
$$

Where $\lambda_{\mathrm{m}, \mathrm{n}}=\mathrm{n} \mathrm{j}_{\mathrm{m}}{ }^{2}$ are the corresponding eigenvalues.

Let $\mathrm{L}_{2}(\mathrm{I})$ be the linear space of functions that are absolutely square integrable on $\mathrm{I}$ and $<\mathrm{f}, \mathrm{g}>$ denote the inner product defined by, 


$$
<f, \bar{g}>=(f, g)=\iint_{I} f(x, t) \overline{g(x, t)} d x d t .
$$

Thus,

$$
\|f\|_{2}^{2}=<f, \bar{f}>=(f, f)=\iint_{I}|f(x, t)|^{2} d x d t
$$

is the norm on $L_{2}(I)$. Hence

$$
\left(\psi_{m, n}(x, t), \psi_{p, q}(x, t)\right)= \begin{cases}1 & \mathrm{~m}=\mathrm{p}, \mathrm{n}=\mathrm{q} \\ 0 & \text { otherwise }\end{cases}
$$

and $\iint_{I}\left|\psi_{m, n}(x, t)\right|^{2} d x d t=1$ implies that $\psi_{m, n}(x, t) \in L_{2}(I)$ for every $m, n \in N_{0}$.

We define the Lagueree finite Hankel transform of $f(x, t) \in L_{2}(I)$ denoted by $L H[f(x, t)](m, n)=F_{L H}(m, n)$ as,

$$
F_{L H}(m, n)=\left(f(x, t), \psi_{m, n}(x, t)\right)=\iint_{I} f(x, t) \psi_{m, n}(x, t) d x d t .
$$

The following theorem provides the inversion of the transformation defined in (2.66).

Theorem 2.1 : Every $\mathrm{f}(\mathrm{x}, \mathrm{t}) \in \mathrm{L}_{2}$ (I) admits the Fourier-Laguerre-Bessel seriesexpansion

$$
f(x, t)=\sum_{m, n=0}^{\infty}\left(f(x, t), \psi_{m, n}(x, t)\right) \psi_{m, n}(x, t)
$$

where the series converges pointwise on I.

\section{Testing Function Space L-H(I)}

Let $m, n \in N_{0}$ and $\nu \geq \frac{-1}{2}$. We denote by L-H(I) the space all complex valued smooth functions $\phi(x, t)$ defined on I such that for each non negative integers $m, n$ and $k$. i)

$$
l^{k}(\phi)=l^{0}\left(\eta_{x, t}^{k} \phi\right)=\left\{\iint_{I}\left[\eta_{x, t}^{k} \phi(x, t)\right]^{2} d x d t\right\}^{\frac{1}{2}}<\infty
$$

ii)

$$
\left(\eta_{x, t}^{k} \phi, \psi_{m, n}(x, t)\right)=\left(\phi, \eta_{x, t}^{k} \psi_{m, n}(x, t)\right)
$$

Obviously $\mathrm{L}_{2}(\mathrm{I}) \subset \mathrm{L}-\mathrm{H}(\mathrm{I})$. L-H(I) is a linear space and $\mathrm{l}^{\mathrm{k}}$ is a seminorm on $\mathrm{L}-\mathrm{H}(\mathrm{I})$. Moreover $\mathrm{l}^{0}$ is a norm on $\mathrm{L}-\mathrm{H}(\mathrm{I})$. Thus $\mathrm{l}^{\mathrm{k}}, \mathrm{k} \in \mathrm{N}_{0}$ is a countable multinorm on $\mathrm{L}-\mathrm{H}(\mathrm{I})$. Also $\mathrm{L}-\mathrm{H}(\mathrm{I})$ is complete and hence a Frechet space. Thus L-H(I) turns out to be a testing function space.

Lemma 3.1 : Every $\psi_{\mathrm{m}, \mathrm{n}}(\mathrm{x}, \mathrm{t})$ is a member of $\mathrm{L}-\mathrm{H}(\mathrm{I})$.

Proof : For each $\mathrm{k} \in \mathrm{N}_{0}$, from equations (2.2) and (3.1) we have

$$
\begin{aligned}
\left|l^{k}\left[\psi_{m, n}(x, t)\right]\right|^{2} & \leq \iint_{I}\left|\eta_{x, t}^{k} \psi_{m, n}(x, t)\right|^{2} d x d t \\
& \leq\left|\lambda_{m, n}\right|^{2 k} \iint_{I}\left|\psi_{m, n}(x, t)\right|^{2} d x d t \\
& =\left|\lambda_{m, n}\right|^{2 k}<\infty .
\end{aligned}
$$

Next since $\lambda_{\mathrm{m}, \mathrm{n}}$ are real then for $m \neq p, n \neq q$, we have 


$$
\begin{aligned}
\left(\eta_{x, t}^{k} \psi_{m, n}(x, t), \psi_{p, q}(x, t)\right) & =\lambda_{m, n}^{k}\left(\psi_{m, n}(x, t), \psi_{p, q}(x, t)\right) \\
& =0=\lambda_{p, q}^{k}\left(\psi_{m, n}(x, t), \psi_{p, q}(x, t)\right) \\
& =\left(\psi_{m, n}(x, t), \lambda_{p, q}^{k} \psi_{p, q}(x, t)\right) \\
& =\left(\psi_{m, n}(x, t), \eta_{x, t}^{k} \psi_{p, q}(x, t)\right)
\end{aligned}
$$

and for $\mathrm{m}=\mathrm{p}, \mathrm{n}=\mathrm{q}$

$$
\begin{aligned}
\left(\eta_{x, t}^{k} \psi_{m, n}(x, t), \psi_{m, n}(x, t)\right) & \\
& =\left(\lambda_{m, n}^{k} \psi_{m, n}(x, t), \psi_{m, n}(x, t)\right) \\
& =\left(\psi_{m, n}, \lambda_{m, n}^{k} \psi_{m, n}(x, t)\right) \\
& =\left(\psi_{m, n}(x, t), \eta_{x, t}^{k} \psi_{m, n}(x, t)\right) .
\end{aligned}
$$

Hence $\psi_{\mathrm{m}, \mathrm{n}}(\mathrm{x}, \mathrm{t}) \in \mathrm{L}-\mathrm{H}(\mathrm{I})$ for all $\mathrm{m}, \mathrm{n} \in \mathrm{N}_{0}$.

Lemma 3.2 : Every $\varphi(\mathrm{x}, \mathrm{t}) \in \mathrm{L}-\mathrm{H}(\mathrm{I})$ can be expanded into the series

$$
\phi(x, t)=\sum_{m=0}^{\infty} \sum_{n=0}^{\infty}\left(\phi(x, t), \psi_{m, n}(x, t)\right) \psi_{m, n}(x, t)
$$

where the series converges in $\mathrm{L}-\mathrm{H}(\mathrm{I})$.

Proof : Let $\varphi(x, t) \in L-H(I)$, then $\eta_{x, t}{ }^{k} \varphi(x, t) \in L_{2}(I)$ and from theorem (2.1), we have

$$
\begin{aligned}
\eta_{x, t}^{k} \phi(x, t) & =\sum_{m=0}^{\infty} \sum_{n=0}^{\infty}\left(\eta_{x, t}^{k} \phi(x, t), \psi_{m, n}(x, t)\right) \psi_{m, n}(x, t) \\
& =\sum_{m=0}^{\infty} \sum_{n=0}^{\infty}\left(\phi, \eta_{x, t}^{k} \psi_{m, n}(x, t)\right) \psi_{m, n}(x, t) \\
& =\sum_{m=0}^{\infty} \sum_{n=0}^{\infty}\left(\phi, \psi_{m, n}(x, t)\right) \lambda_{m, n}^{k} \psi_{m, n}(x, t) \\
& =\sum_{m=0}^{\infty} \sum_{n=0}^{\infty}\left(\phi, \psi_{m, n}(x, t)\right) \eta^{k} \psi_{m, n}(x, t)
\end{aligned}
$$

which implies that $l^{k}\left[\phi(x, t)-\sum_{m=0}^{M} \sum_{n=0}^{N}(\phi(x, t), \psi(x, t)) \psi(x, t)\right] \rightarrow 0$

as $M \rightarrow \infty, N \rightarrow \infty$ independently. Thus the series in (3.3) converges to $\phi(x, t)$ in $\mathrm{L}-\mathrm{H}(\mathrm{I})$.

\section{The Generalized Function Space L-H'(I)}

The space of all continuous linear functionals on L-H(I), denoted by L-H'(I) is called the dual of L$\mathrm{H}(\mathrm{I})$ and members of L-H'(I) are called generalized functions on I. The number that $\mathrm{f} \in \mathrm{L}-\mathrm{H}^{\prime}(\mathrm{I})$ assigns to $\varphi \in$ $\mathrm{L}-\mathrm{H}(\mathrm{I})$ is denoted by $\langle\mathrm{f}, \varphi>$. Since the testing function space L-H(I) is complete, so also is L- H'(I)[5] Let $\mathrm{f}(\mathrm{x}, \mathrm{t})$ be a real valued continuous function locally integrable on I such that

$$
\iint_{I}|f(x, t)|^{2} d x d t<\infty
$$

thenf generates a member of L-H'(I) through the definition

$$
<f, \phi>=\iint_{I} f(x, t) \phi(x, t) d x d t .
$$

Clearly (4.1) defines a linear functional $\mathrm{f}$ on $\mathrm{L}-\mathrm{H}(\mathrm{I})$ and the continuity of $\mathrm{f}$ can be verified by using Schwarz's inequality. Such members of L-H'(I) are called regulargeneralized functions in L-H'(I). All other generalized functions in L-H'(I) are called singular generalized functions.

Now we define a generalized differential operator $\eta_{x, t}$ on L-H'(I) through the relationship 


$$
\left(f, \eta_{x, t} \phi\right)=<f, \overline{\eta_{x, t} \phi}>=<\overline{\eta_{x t}} f, \bar{\phi}>=\left(\overline{\eta_{x, t}} f, \phi\right)
$$

where $\overline{\eta_{x, t}}$ is obtained from $\eta_{\mathrm{x}, \mathrm{t}}$ by reversing the order in which the differentiation and multiplication by smooth functions occurring in $\eta_{x, t}$, replacing each $\mathrm{D}$ by $-\mathrm{D}$ and then taking the complex conjugate of the result.

But this is precisely the same expression for $\eta_{\mathrm{x}, \mathrm{t}}[5]$. Thus $\eta_{\mathrm{x}, \mathrm{t}}={\overline{\eta_{x, t}}}^{\prime}$ is defined as the generalized differential operator on L-H'(I) through the equation

$<\eta_{\mathrm{x}, \mathrm{f}} \mathrm{f}, \varphi>=<\mathrm{f}, \eta_{\mathrm{x}, \mathrm{t}} \varphi>$

wheref $\in \mathrm{L}-\mathrm{H}^{\prime}(\mathrm{I}), \varphi \in \mathrm{L}-\mathrm{H}(\mathrm{I})$.

Some properties of L-H(I) and L-H'(I):

I) $\mathrm{D}(\mathrm{I}) \subset \mathrm{L}-\mathrm{H}(\mathrm{I}) \subset \mathrm{E}(\mathrm{I})$ and since $\mathrm{D}(\mathrm{I})$ is dense in $\mathrm{E}(\mathrm{I}), \mathrm{L}-\mathrm{H}(\mathrm{I})$ is also dense in $\mathrm{E}(\mathrm{I})$. It follows $\mathrm{E}^{\prime}(\mathrm{I})$ is a subspace of L-H'(I). The convergence of a sequence in $\mathrm{D}(\mathrm{I})$ implies its convergence in L-H(I). Therestriction of any $\mathrm{f} \in$ L-H(I) to D(I) is in D'(I). Moreover the convergence in L-H'(I)implies convergence in D'(I).

II) For each $\mathrm{f} \in \mathrm{L}-\mathrm{H}$ '(I) there exists a non-negativeinteger $\mathrm{r}$ and a positive constant $\mathrm{C}$ such that

$$
|<f, \phi>| \leq C \max _{0 \leq k \leq r} l^{k}(\phi)
$$

for every $\varphi \in \mathrm{L}-\mathrm{H}(\mathrm{I})$. Here $\mathrm{r}$ and $\mathrm{C}$ depends on $\mathrm{f}$ but not on $\varphi$.

III) The mapping $\varphi \rightarrow \eta_{x, t} \varphi$ is continuous linear mapping of L-H(I) into itself. It follows thatf $\rightarrow \eta_{x, f}$ fis also a continuous linear mapping of L-H'(I) whenever $\mathrm{f}$ is a regular generalizedfunction in $\mathrm{L}-\mathrm{H}^{\prime}(\mathrm{I})$.

\section{Lagueree-Finite Hankel Transformation of Generalized Functions} $=\mathrm{F}_{\mathrm{LH}}(\mathrm{m}, \mathrm{n})$ as,

We definetheLagueree-Finite HankelTransform of generalized function $f \in L-H^{\prime}(I)$, denoted by LH[f ]

Where $\psi_{\mathrm{m}, \mathrm{n}}(\mathrm{x}, \mathrm{t}) \in \mathrm{L}-\mathrm{H}(\mathrm{I})$ for $\mathrm{m}, \mathrm{n} \in \mathrm{N}_{0}$.

$$
\operatorname{LH}[f(x, t)](m, n)=F_{L H}(m, n)=\left(f(x, t), \psi_{m, n}(x, t)\right)
$$

We see that LH is a linear and continuous mapping on L-H'(I) which maps $f \in L_{-}-H^{\prime}(I)$ into a function $F_{L H}(m, n)$ defined on $\mathrm{N}_{0} \times \mathrm{N}_{0}$. The following theorem provides the inversion of the transformation defined in (5.1).

Theorem 5.1: Let $\mathrm{f} \in \mathrm{L}-\mathrm{H}^{\prime}(\mathrm{I})$, then the series

$$
\sum_{m=0}^{\infty} \sum_{n=0}^{\infty}\left(f(x, t), \psi_{m, n}(x, t)\right) \psi_{m, n}(x, t)
$$

converges to $\mathrm{f}$ in $\mathrm{L}-\mathrm{H}^{\prime}(\mathrm{I})$.

Proof: From lemma 3.2 we have for every $\phi \in \mathrm{L}-\mathrm{H}(\mathrm{I})$, the series $\sum_{m=0}^{\infty} \sum_{n=0}^{\infty}\left(\phi, \psi_{m, n}(x, t)\right) \psi_{m, n}(x, t)$ converges to $\phi$ in L-H(I), then for $f \in \mathrm{L}^{-} \mathrm{H}^{\prime}(\mathrm{I})$, we write

$$
\begin{aligned}
(f, \phi) & =\left(f, \sum_{m=0}^{\infty} \sum_{n=0}^{\infty}\left(\phi, \psi_{m, n}(x, t)\right), \psi_{m, n}(x, t)\right) \\
& =\sum_{m=0}^{\infty} \sum_{n=0}^{\infty} \overline{\left(\phi, \psi_{m, n}(x, t)\right)}\left(f, \psi_{m, n}(x, t)\right) \\
& =\sum_{m=0}^{\infty} \sum_{n=0}^{\infty}\left(f, \psi_{m, n}(x, t)\right)\left(\psi_{m, n}(x, t), \phi(x, t)\right.
\end{aligned}
$$




$$
\begin{aligned}
& =\sum_{m=0}^{\infty} \sum_{n=0}^{\infty}\left(\left(f, \psi_{m, n}(x, t)\right) \psi_{m, n}(x, t), \phi(x, t)\right) \\
& =\left(\sum_{m=0}^{\infty} \sum_{n=0}^{\infty}\left(f, \psi_{m, n}(x, t)\right) \psi_{m, n}(x, t), \phi(x, t)\right)
\end{aligned}
$$

Thus the series $\sum_{m=0}^{\infty} \sum_{n=0}^{\infty}\left(f, \psi_{m, n}(x, t)\right) \psi_{m, n}(x, t)$ converges weakly to $f$ in L-H'(I). The above theorem lead to define the inverse of the Laguerre-finite Hankel transformation of $\mathrm{f} \in \mathrm{L}-\mathrm{H}$ '(I), denoted by $\mathrm{LH}^{-1} \mathrm{~F}_{\mathrm{LH}}(\mathrm{m}, \mathrm{n})=\mathrm{f}(\mathrm{x}, \mathrm{t})$, as

$$
\begin{aligned}
\mathcal{L H}^{-1} \mathcal{F}_{L H}(m, n)=f(x, t) & =\sum_{m=0}^{\infty} \sum_{n=0}^{\infty} \mathcal{F}_{L H}(m, n) \psi_{m, n}(x, t) \\
& =\sum_{m=0}^{\infty} \sum_{n=0}^{\infty}\left(f(x, t), \psi_{m, n}(x, t)\right) \psi_{m, n}(x, t)
\end{aligned}
$$

Theorem 5.2 (Uniqueness Theorem): Let $f, g \in L-H^{\prime}(I)$ are such that

$$
\mathrm{LH}[\mathrm{f}](\mathrm{m}, \mathrm{n})=\mathrm{F}_{\mathrm{LH}}(\mathrm{m}, \mathrm{n})=\mathrm{G}_{\mathrm{LH}}(\mathrm{m}, \mathrm{n})=\mathrm{LH}[\mathrm{g}](\mathrm{m}, \mathrm{n})
$$

for every $m, n \in N_{0}$, then $f=g$ in the sense of equality in L-H'(I).

Proof : Let $\varphi \in \mathrm{L}-\mathrm{H}(\mathrm{I})$, and $\mathrm{f}, \mathrm{g} \in \mathrm{L}-\mathrm{H}^{\prime}(\mathrm{I})$ then

$$
\begin{aligned}
& <\mathrm{f}, \phi>-<\mathrm{g}, \phi>=<\sum_{\mathrm{m}=0}^{\infty} \sum_{\mathrm{n}=0}^{\infty}\left(\mathrm{f}, \psi_{\mathrm{m}, \mathrm{n}}(\mathrm{x}, \mathrm{t})\right) \psi_{\mathrm{m}, \mathrm{n}}(\mathrm{x}, \mathrm{t}), \phi(\mathrm{x}, \mathrm{t})> \\
& \text { - } \quad<\sum_{\mathrm{m}=0}^{\infty} \sum_{\mathrm{n}=0}^{\infty}\left(\mathrm{g}, \Psi_{\mathrm{m}, \mathrm{n}}(\mathrm{x}, \mathrm{t})\right) \Psi_{\mathrm{m}, \mathrm{n}}(\mathrm{x}, \mathrm{t}), \phi(\mathrm{x}, \mathrm{t})> \\
& =<\sum_{\mathrm{m}=0}^{\infty} \sum_{\mathrm{n}=0}^{\infty} \mathrm{F}_{\mathrm{LH}}(\mathrm{m}, \mathrm{n}) \Psi_{\mathrm{m}, \mathrm{n}}(\mathrm{x}, \mathrm{t}), \phi(\mathrm{x}, \mathrm{t})> \\
& \text { - } \quad<\sum_{\mathrm{m}=0}^{\infty} \sum_{\mathrm{n}=0}^{\infty} \mathrm{G}_{\mathrm{LH}}(\mathrm{m}, \mathrm{n}) \psi_{\mathrm{m}, \mathrm{n}}(\mathrm{x}, \mathrm{t}), \phi(\mathrm{x}, \mathrm{t})> \\
& =\left\langle\sum_{\mathrm{m}=0}^{\infty} \sum_{\mathrm{n}=0}^{\infty}\left[\mathrm{F}_{\mathrm{LH}}(\mathrm{m}, \mathrm{n})-\mathrm{G}_{\mathrm{LH}}(\mathrm{m}, \mathrm{n})\right] \psi_{\mathrm{m}, \mathrm{n}}(\mathrm{x}, \mathrm{t}), \phi(\mathrm{x}, \mathrm{t})\right\rangle \\
& =0 \text {. }
\end{aligned}
$$

\section{An Operational Calculus}

Let $f(x, t) \in L-H^{\prime}(I), \psi_{m, n}(x, t) \in L-H(I)$ and since the differential operator $\eta_{x, t}$ is a continuous linear mapping of L-H'(I) into itself, then from equation (4.3), we have

$$
\begin{aligned}
& \operatorname{LH}\left[\eta_{x, t}{ }^{k} f\right](m, n)=<\eta_{x, t}{ }^{k} f, \psi_{m, n}(x, t)>=<f, \eta_{x, t}{ }^{k} \psi_{m, n}(x, t)> \\
& =<f, \lambda_{m, n}{ }^{k} \psi_{m, n}(x, t)> \\
& =\lambda_{m, n}{ }^{k}<f, \psi_{m, n}(x, t)> \\
& =\lambda_{m, n}{ }^{k} L H[f](m, n) \\
& =\lambda_{m, n}{ }^{k} F_{L H}(m, n) .
\end{aligned}
$$

We can use this fact to solve the distributional differential equations of the form

$$
P\left(\eta_{x, t}\right) u=g
$$

whereP is a polynomial and the given $g$ and unknown $u$ are the generalized functions in L-H'(I). Applying the Laguerre Finite Hankel transformation defined in (5.1) to the differential equation (6.2), we get

$$
\mathrm{P}\left(\lambda_{\mathrm{m}, \mathrm{n}}\right) \mathrm{LH}[\mathrm{u}](\mathrm{m}, \mathrm{n})=\mathrm{LH}[\mathrm{g}](\mathrm{m}, \mathrm{n}), \mathrm{m}, \mathrm{n} \in \mathrm{N}_{0} .
$$

If $\mathrm{P}\left(\lambda_{\mathrm{m}, \mathrm{n}}\right) \neq 0$ for all $\mathrm{m}, \mathrm{n} \in \mathrm{N}_{0}$, we divide (6.3) by $\mathrm{P}\left(\lambda_{\mathrm{m}, \mathrm{n}}\right)$ and apply inverse Laguerre Finite Hankel transform defined in (5.3), and get 


$$
u(x, t)=\sum_{m=0}^{\infty} \sum_{n=0}^{\infty} \frac{\mathcal{L} \mathcal{H}[g](m, n)}{P\left(\lambda_{m, n}\right)} \psi_{m, n}(x, t)
$$

where the series converges in L-H'(I). In view of Theorem (5.1) and (5.2) the solution $\mathrm{u}(\mathrm{x}, \mathrm{t})$ in $\mathrm{L}-\mathrm{H}^{\prime}(\mathrm{I})$ exists and is unique.

\section{References}

[1] A. H. Zemanian, Orthonormal series expansions of certain distributions and distributional transform calculus, J. Math. Anal. Appl. 14 (1966), 263-275.

[2] S. D. Bhosale and S. V. More, On Marchi-Zgrablich transformation of generalized functions, IMA J. Appl. Maths. 33 (1984), $33-$ 42 .

[3] S. K. Panchal and S. V. More, On modified Marchi-Zgrablich transformation of generalized functions, J. Indian Acad. Math 17(1) (1995), 13-26.

[4] S. K. Panchal, Laguerre-Finite Hankel Transform of Generalized Functions, Proceedings of the International Conference on Mathematical Sciences in honour of Professor A.M. Mathai, St. Thomas College Pala, Kottayam, Kerala, India.(Jan 3-5, 2011).

[5] A. H. Zemanian Generalized Integral Transformations, Interscience Publisher, New York, 1968. 